\title{
Plasma Mesothelin as a Novel Diagnostic and Prognostic Biomarker in Colorectal Cancer
}

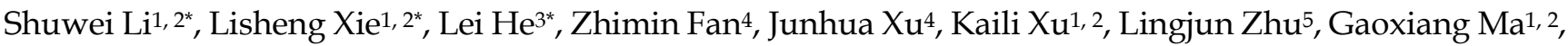

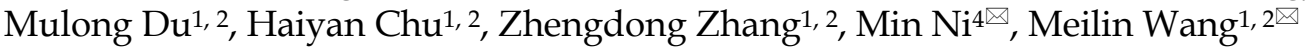 \\ 1. Department of Environmental Genomics, Jiangsu Key Laboratory of Cancer Biomarkers, Prevention and Treatment, Collaborative Innovation Center for \\ Cancer Personalized Medicine, Nanjing Medical University, Nanjing, China; \\ 2. Department of Genetic Toxicology, The Key Laboratory of Modern Toxicology of Ministry of Education, School of Public Health, Nanjing Medical \\ University, Nanjing, China; \\ 3. First School of Clinical Medicine, Nanjing University of Chinese Medicine, Nanjing, China; \\ 4. Department of Colorectal Surgery, The Third Affiliated Hospital of Nanjing University of Chinese Medicine, Nanjing, China; \\ 5. Department of Oncology, The First Affiliated Hospital of Nanjing Medical University, Nanjing, China. \\ * These authors contributed equally to the writing of this article.
}

$\triangle$ Corresponding author: Meilin Wang, Ph.D, School of Public Health, Nanjing Medical University 818 East Tianyuan Road, Jiangning District, Nanjing, Jiangsu, 211166, P. R. China; Tel: +86-25-86868417; e-mail: mwang@njmu.edu.cn or Min Ni, Department of Colorectal Surgery, The Third Affiliated Hospital of Nanjing University of Chinese Medicine, Nanjing 210001, P. R. China; e-mail: niminnj@163.com

(C) Ivyspring International Publisher. This is an open access article distributed under the terms of the Creative Commons Attribution (CC BY-NC) license (https://creativecommons.org/licenses/by-nc/4.0/). See http://ivyspring.com/terms for full terms and conditions.

Received: 2016.10.20; Accepted: 2017.02.27; Published: 2017.05.12

\begin{abstract}
Objective Mesothelin is a cell surface protein and overexpressed in many cancers. However, the potential value of mesothelin as plasma biomarker in colorectal cancer has not been explored. The purpose of this study was to identify whether plasma mesothelin is a suitable diagnostic and prognostic biomarker for colorectal cancer.

Methods We performed a two-stage case-control study to evaluate plasma mesothelin levels in colorectal cancer using enzyme-linked immunosorbent assay (ELISA). Preoperative and postoperative plasma were collected to examine the level changes influenced by surgery. Receiver operating characteristic (ROC) curves were applied to identify the diagnostic value of plasma mesothelin. We also conducted univariate Kaplan-Meier survival analysis and Cox regression analysis of patients with survival information.

Results We found that the plasma mesothelin levels in colorectal cancer patients were significantly higher than that in the controls $(P<0.001)$ with an AUC value of $0.690(95 \% \mathrm{Cl}=0.625$ to 0.752$)$. Individuals with lower mesothelin level had a longer survival time (adjusted $\mathrm{HR}=4.43,95 \% \mathrm{Cl}=$ 1.93-10.15, $P<0.001$ ). Furthermore, Patients had slightly decreased mesothelin levels in postoperative plasma than preoperative plasma, although the alteration was not statistically significant $(P=0.052)$.

Conclusion Our findings highlight the correlative relationship between plasma mesothelin levels and the presence and progression of colorectal cancer. Plasma mesothelin may be a potential diagnostic and, or prognostic biomarker for colorectal cancer.
\end{abstract}

Key words: mesothelin, colorectal cancer, biomarker.

\section{Introduction}

Colorectal cancer is the third most common malignant tumor by incidence around the world [1]. About 134,490 new cases will be diagnosed with colorectal cancer in 2016, estimated by the American Cancer Society [2]. Many classical colorectal cancer screening tests have been optional and applied for decades, including fecal occult blood tests (FOBT), endoscopic and radiologic examinations $[3,4]$. However, nearly $25 \%$ patients with colorectal cancer were diagnosed advancing stage at the first time, and the 5-year survival rate of colorectal cancer was estimated to be $62 \%-64 \%[5,6]$. Due to the limitation of these screening tests in the early diagnosis of colorectal cancer, it is necessary to 
identify new potential biomarkers, especially in plasma or serum, for the diagnosis of colorectal cancer and prediction of therapy.

Mesothelin, a 40-kDa cell glycosylphosphatidyl inositol (GPI)-linked protein, has been found in mesothelial cells lining the peritoneum, pleura and pericardium usually $[7,8]$. The overexpressed mesothelin has been detected in mesothelioma and pancreatic cancer $[9,10]$. Mesothelin overexpression has also be found in many other cancers in serum and other body fluids, including ovarian malignancies, gastric cancer and lung adenocarcinomas [11-13]. Recent studies have reported the biological function of mesothelin with colorectal cancer in vitro [14]. Moreover, the significantly overexpressed mesothelin was identified in colon cancer serum [15]. No studies to date are available about the plasma mesothelin as potential biomarker for colorectal cancer.

In this study, we investigated the level of mesothelin in plasma in a two-stage case-control study and evaluated the diagnosis and prognosis value of colorectal cancer patients with high mesothelin level. Moreover, we estimated the difference of mesothelin level between preoperative plasma and postoperative plasma samples.

\section{Methods}

\section{Study participants}

A total of 147 patients with colorectal cancer and 121 healthy controls were recruited in this study. In the first stage, 40 primary colorectal cancer cases were pathologically confirmed in the Third Affiliated Hospital of Nanjing University of Chinese Medicine before May 2016. Forty cancer-free controls were recruited from those seeking medical care in local hospitals with frequency-matched to cases on age $( \pm 5$ years) and gender. In the second stage, 107 colorectal cancer cases and 81 controls were recruited from the First Affiliated Hospital and the Affiliated Nanjing First Hospital of Nanjing Medical University starting from September 2010. A total of 54 patients were followed-up through telephone calls at regular intervals for up to 5 years and the median survival time (MST) was 41.3 months. Approval for this research was acquired from the Institutional Review Board of Nanjing Medical University.

\section{Preparation of plasma}

A total of $5 \mathrm{ml}$ blood of all subjects was collected in anticoagulant tubes and centrifuged to obtain plasma samples. Additionally, a paired-sample set of preoperative plasma and postoperative plasma (7 days after postsurgical removal of primary tumors) were collected from 9 colorectal cancer patients. All the samples were stored at $-80^{\circ} \mathrm{C}$ for further experiment.

\section{Plasma mesothelin detection}

To determine the level of mesothelin in plasma samples, a human enzyme-linked immunosorbent assay (ELISA) kit (Cusabio Biotech Co., Ltd., Wuhan, China) was performed following the manufacturer's instructions. All samples were assayed after 5-fold dilution and the tests were conducted at least in duplicate. Moreover, the investigators were blind to participant clinical status. The lower limit of detection of mesothelin was $0.078 \mathrm{ng} / \mathrm{ml}$ and all the samples were measured over the limit.

\section{Statistical analysis}

We conducted Student's $t$-test and $\chi^{2}$ test to give the $P$ value to evaluate the differences of demographic characteristics between colorectal cases and controls. The level of mesothelin was showed as mean \pm standard deviation (SD). We used Wilcoxon test to compare the statistical differences in plasma mesothelin level between two groups. The analysis method was also applied to estimate the statistical differences between preoperative plasma and postoperative plasma samples. To identify the diagnostic power of plasma mesothelin, we used receiver operating characteristic (ROC) curves and the area under the curve (AUC). The suitable diagnostic cutoff value of mesothelin was determined by Youden index [16]. To evaluate the association between mesothelin level and the risk of colorectal cancer, logistic regression analyses were performed to calculate odds ratios (ORs) and their 95\% confidence intervals (CIs). Moreover, log-rank test was used to analyze the subgroup's survival time. Hazard ratios (HRs) and their 95\% CIs were calculated by cox regression analysis. Two-sided $P$-value less than 0.05 was considered as statistically significance and all the statistical analyses were conducted with SAS software (SAS Institute, Cary, NC, USA).

\section{Results}

\section{Study population characteristics}

There were 147 colorectal cancer cases and 121 cancer-free controls in this two-stage case-control study. The detailed information of characteristics is shown in Table 1 . The average age of cases and controls was 60.7 and 59.6 respectively, and no significant differences between patients and controls were identified in age $(P=0.500)$ and sex $(P=0.858)$. In addition, we found no notable differences in drinking and smoking status between two groups $(P>$ $0.05)$. However, significantly increased frequency of family history of cancers was found in colorectal cancer group $(P<0.001)$. 
Table 1. Characteristics and clinical features between colorectal cancer cases and controls

\begin{tabular}{|c|c|c|c|c|c|c|c|c|c|}
\hline \multirow[t]{2}{*}{ Variables } & \multicolumn{3}{|l|}{ Stage 1} & \multicolumn{3}{|l|}{ Stage 2} & \multicolumn{3}{|l|}{ Combineda $^{\mathrm{a}}$} \\
\hline & Cases, N (\%) & Control, N (\%) & $P$ & Cases, N (\%) & Control, N (\%) & $P$ & Cases, N (\%) & Control, N (\%) & $P$ \\
\hline Age (mean \pm SD) & $60.6 \pm 13.8$ & $59.2 \pm 15.3$ & 0.673 & $60.7 \pm 12.0$ & $59.7 \pm 14.5$ & 0.611 & $60.7 \pm 12.5$ & $59.5 \pm 14.7$ & 0.500 \\
\hline \multicolumn{10}{|l|}{ Sex } \\
\hline Male & $23(57.5 \%)$ & $22(55.0 \%)$ & 0.822 & $60(56.1 \%)$ & $45(55.6 \%)$ & 0.943 & $83(56.5 \%)$ & $67(55.4 \%)$ & 0.858 \\
\hline Female & $17(42.5 \%)$ & $18(45.0 \%)$ & & $47(43.9 \%)$ & $36(44.4 \%)$ & & $64(43.5 \%)$ & $54(44.6 \%)$ & \\
\hline \multicolumn{10}{|l|}{ Smoking status } \\
\hline Never & $27(67.5 \%)$ & $26(76.0 \%)$ & 0.813 & $77(72.0 \%)$ & $66(81.5 \%)$ & 0.130 & $104(70.8 \%)$ & $92(76.0 \%)$ & 0.331 \\
\hline Ever & $13(32.5 \%)$ & $14(24.0 \%)$ & & $30(28.0 \%)$ & $15(18.5 \%)$ & & $43(29.2 \%)$ & $29(24.0 \%)$ & \\
\hline \multicolumn{10}{|l|}{ Drinking status } \\
\hline Never & $25(62.5 \%)$ & $31(77.5 \%)$ & 0.143 & $81(75.7 \%)$ & $63(77.8 \%)$ & 0.740 & $106(72.1 \%)$ & $94(77.7 \%)$ & 0.296 \\
\hline Ever & $15(37.5 \%)$ & $9(22.5 \%)$ & & $26(24.3 \%)$ & $18(22.2 \%)$ & & $41(27.9 \%)$ & $27(22.3 \%)$ & \\
\hline \multicolumn{10}{|l|}{$\mathrm{FH}$} \\
\hline No & $29(72.5 \%)$ & $32(80.0 \%)$ & 0.431 & $81(75.7 \%)$ & $78(96.3 \%)$ & $<0.001$ & $110(74.8 \%)$ & $110(90.9 \%)$ & $<0.001$ \\
\hline Yes & $11(27.5 \%)$ & $8(20.0 \%)$ & & $26(24.3 \%)$ & $3(3.7 \%)$ & & $37(25.2 \%)$ & $11(9.1 \%)$ & \\
\hline \multicolumn{10}{|l|}{ Tumor site } \\
\hline Colon & $19(47.5 \%)$ & & & $54(50.5 \%)$ & & & $73(49.7 \%)$ & & \\
\hline Rectal & $21(52.5 \%)$ & & & $53(49.5 \%)$ & & & $74(50.3 \%)$ & & \\
\hline \multicolumn{10}{|l|}{ Tumor size } \\
\hline$\leq 4.0 \mathrm{~cm}$ & $19(65.5 \%)$ & & & $52(58.4 \%)$ & & & $71(60.2 \%)$ & & \\
\hline$>4.0 \mathrm{~cm}$ & $10(34.5 \%)$ & & & $37(41.6 \%)$ & & & $47(39.8 \%)$ & & \\
\hline \multicolumn{10}{|l|}{ Tumor grade } \\
\hline Low & $4(10.0 \%)$ & & & $5(5.1 \%)$ & & & $9(6.1 \%)$ & & \\
\hline Intermediate & $32(80.0 \%)$ & & & $79(73.8 \%)$ & & & $111(75.5 \%)$ & & \\
\hline High & $4(10.0 \%)$ & & & $23(21.5 \%)$ & & & $27(18.4 \%)$ & & \\
\hline \multicolumn{10}{|l|}{ Duke's stage } \\
\hline A & $4(10.0 \%)$ & & & $8(7.5 \%)$ & & & $12(8.2 \%)$ & & \\
\hline B & $17(42.5 \%)$ & & & $45(42.0 \%)$ & & & $62(42.2 \%)$ & & \\
\hline $\mathrm{C}$ & $14(35.0 \%)$ & & & $35(32.7 \%)$ & & & $49(33.3 \%)$ & & \\
\hline $\mathrm{D}$ & $5(12.5 \%)$ & & & $19(17.8 \%)$ & & & $24(16.3 \%)$ & & \\
\hline
\end{tabular}

aCombine Stage 1 and Stage 2 as the combined stage.

$\mathrm{SD}$, standard deviation.

$\mathrm{FH}$, family history of cancers

\section{Preoperative plasma mesothelin levels in cases and controls}

We used ELISA to estimate mesothelin levels in all preoperative plasma samples. In stage 1 , we used plasma samples of 40 colorectal cancer cases and 40 cancer-free controls to explore the mesothelin levels in different groups. We found that the median levels of mesothelin in colorectal cancer patients and controls were $32.95 \mathrm{ng} / \mathrm{ml}$ and $20.31 \mathrm{ng} / \mathrm{ml}$, respectively. A significantly higher level of mesothelin in the cases was identified compared with that in the controls $(P<$ 0.001). Subsequently, we estimated the mesothelin levels in additional samples in stage 2 . The median mesothelin level in patients $(35.12 \mathrm{ng} / \mathrm{ml})$ was significantly higher than that in control group (27.27 $\mathrm{ng} / \mathrm{ml})(P<0.001)$. Combined the results in stage 1 and stage 2 , we obtained the consistent results that colorectal cancer patients had more mesothelin in plasma than that in the controls (median level $=34.68$ $\mathrm{ng} / \mathrm{ml}$ for cases; median level $=25.65 \mathrm{ng} / \mathrm{ml}$ for controls; $P<0.001$ ) (Figure 1).

To evaluate plasma mesothelin levels of cases according to the demographic and clinical variables, we compared the levels of mesothelin under different subgroups. As shown in Table 2, there were no significant differences in plasma mesothelin levels between demographic subgroups, including sex, age, family history of cancers, drinking and smoking status. However, we found that the mesothelin levels in group of Duke's stage C and D were significantly higher than the group of Duke's stage A and B $(P<$ 0.001 , Figure 1). According to tumor grade, site and size, no significant differences were observed in the mesothelin levels.

\section{The prediction and diagnosis of colorectal cancer using plasma mesothelin}

ROC analysis was conducted to obtain the performance of plasma mesothelin levels in the diagnosis of colorectal cancer. We identified the accuracy of plasma mesothelin levels to discriminate colorectal cancer patients from controls with an AUC value of 0.69 (95\% CI $=0.63-0.75)$ (data not shown). To identify a patient with colorectal cancer using plasma mesothelin, the sensitivity and specificity were $48.3 \%$ and $83.5 \%$, respectively. The value of Youden index was $36.50 \mathrm{ng} / \mathrm{ml}$ according to sensitivity and specificity. 
Table 2. Preoperative plasma mesothelin of colorectal cancer cases in subgroups.

\begin{tabular}{llll}
\hline Variables & Cases, N & Mesothelin $(\mathrm{ng} / \mathrm{ml})$ & Pa \\
\hline Age (years) & $73(49.7 \%)$ & $35.72 \pm 16.01$ & 0.800 \\
$\leq 60$ & $74(50.3 \%)$ & $35.95 \pm 16.12$ & \\
$>60$ & & & \\
Sex & $83(56.5 \%)$ & $35.07 \pm 14.88$ & 0.618 \\
$\quad$ Male & $64(43.5 \%)$ & $36.82 \pm 17.44$ & \\
Female & & & 0.723 \\
Smoking status & $104(70.8 \%)$ & $35.50 \pm 16.00$ & \\
Never & $43(29.2 \%)$ & $36.64 \pm 16.19$ & 0.981 \\
Ever & & & \\
Drinking status & $106(72.1 \%)$ & $35.97 \pm 16.32$ & 0.427 \\
Never & $41(27.9 \%)$ & $35.48 \pm 15.37$ & \\
Ever & & & \\
FH & $110(74.8 \%)$ & $35.13 \pm 15.54$ & 0.455 \\
No & $37(25.2 \%)$ & $37.93 \pm 17.38$ & \\
Yes & & & \\
Tumor site & $73(49.7 \%)$ & $34.87 \pm 15.75$ & \\
Colon & $74(50.3 \%)$ & $36.79 \pm 16.32$ & \\
Rectal & & & \\
Tumor size & $71(60.2 \%)$ & $35.85 \pm 17.14$ & \\
$\leq 4.0$ cm & $47(39.8 \%)$ & $34.76 \pm 14.60$ & \\
$>4.0$ cm & $120(81.6 \%)$ & $34.90 \pm 16.39$ & \\
Tumor grade & $27(18.4 \%)$ & $40.01 \pm 13.68$ & \\
Low + Intermediate & & & \\
High & & $30.47 \pm 13.38$ & \\
Duke's stage & & & \\
A + B & & & \\
C + D & & & \\
\hline
\end{tabular}

aTwo-sided Wilcoxon test for statistical differences between different groups FH, family history of cancers
We further used multivariable logistic analysis to estimate the risk of colorectal cancer and high level of mesothelin in plasma. We found that individuals with higher plasma mesothelin level $(>36.50 \mathrm{ng} / \mathrm{ml})$ were significantly associated with the risk of colorectal cancer compared to those with lower plasma mesothelin level $(\leq 36.50 \mathrm{ng} / \mathrm{ml})(\mathrm{OR}=4.76$, 95\% CI $=2.61-8.65, P<0.001)$ (Table 3). Moreover, significantly increased colorectal cancer risk was also observed in participants with family history of cancers $(\mathrm{OR}=3.29,95 \% \mathrm{CI}=1.54-7.04, P=0.002)$.

\section{The utilization of plasma mesothelin in the operative effect evaluation}

We collected 9 paired preoperative plasma and postoperative plasma samples from colorectal cancer subjects and tested the mesothelin levels. Plasma mesothelin levels were found to be slightly decreased after surgical resection of tumors $(P=0.052)$ (Figure S1).

Table 3. Multivariable logistic analysis for plasma mesothelin and various diagnostic factors in patients with colorectal cancer.

\begin{tabular}{lll}
\hline Variables & OR $(95 \% \mathrm{CI})$ & $P^{c}$ \\
\hline Age, $>60$ y $v s . \leq 60 y^{\text {a }}$ & $0.99(0.58$ to 1.70$)$ & 0.966 \\
Sex, female $v s$. male & $0.88(0.51$ to 1.52$)$ & 0.640 \\
Smoking status, ever $v$ s. never & $1.17(0.57$ to 2.42$)$ & 0.673 \\
Drinking status, ever $v$ s. never & $1.44(0.68$ to 3.04$)$ & 0.347 \\
FH, yes $v$ s. no & $3.29(1.54$ to 7.04$)$ & 0.002 \\
Plasma mesothelin $(\mathrm{ng} / \mathrm{ml}),>36.50$ vs. $\leq$ & $4.76(2.61$ to 8.65$)$ & $<0.001$ \\
36.50 & & \\
\hline aThe median age was 60 years. & & \\
bThe cutoff values of plasma mesothelin was derived by Youden index. \\
cAdjusted by age, sex, family history of cancers, smoking and drinking status in \\
logistic regression analysis. \\
FH, family history of cancers
\end{tabular}

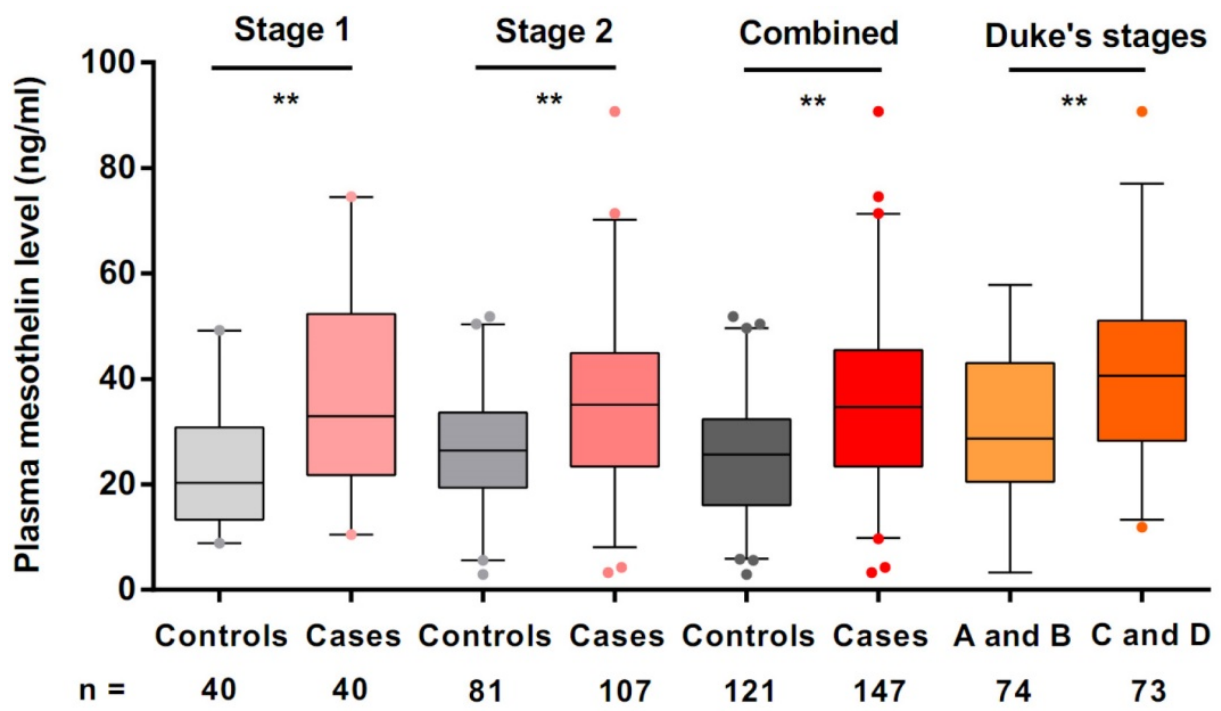

Figure 1. Preoperative plasma mesothelin levels in healthy controls and colorectal cancer patients under different Duke's stages. Boxplots represent plasma mesothelin levels in healthy controls $(n=121)$ and colorectal cancer cases $(n=147)$ and patients under different Duke's stages $(A+B ; n=74, C+$ $\mathrm{D} ; \mathrm{n}=73$ ). Boxes indicate the interquartile range, and median values are shown by the horizontal lines across boxes. Statistically significant differences were determined using two-sided Wilcoxon test. $* * P<0.001$. 
Table 4. Associations between selected variables and overall survival of colorectal cancer.

\begin{tabular}{|c|c|c|c|c|c|c|}
\hline Variables & $\mathrm{N}$ (patients) & $\mathrm{N}$ (deaths) & Median OS (months) & Log-rank $P$ & $\mathrm{HR}(95 \% \mathrm{CI})$ & $P^{\mathrm{b}}$ \\
\hline \multicolumn{7}{|l|}{ Age (years) } \\
\hline$\leq 60$ & 29 & $19(65.5 \%)$ & 44.0 & & 1 & \\
\hline$>60$ & 25 & $19(76.0 \%)$ & 39.3 & 0.402 & $2.83(1.33-6.00)$ & 0.007 \\
\hline \multicolumn{7}{|l|}{ Sex } \\
\hline Male & 30 & $19(63.3 \%)$ & 44.1 & & 1 & \\
\hline Female & 24 & $19(79.2 \%)$ & 39.7 & 0.378 & $1.82(0.79-4.17)$ & 0.159 \\
\hline \multicolumn{7}{|l|}{ Smoking status } \\
\hline Never & 38 & $26(68.4 \%)$ & 43.7 & & 1 & \\
\hline Ever & 16 & $12(75.0 \%)$ & 34.5 & 0.418 & $1.07(0.43-2.69)$ & 0.881 \\
\hline \multicolumn{7}{|l|}{ Drinking status } \\
\hline Never & 40 & $28(70.0 \%)$ & 43.7 & & 1 & \\
\hline Ever & 14 & $10(71.4 \%)$ & 29.6 & 0.357 & $3.72(1.26-11.0)$ & 0.018 \\
\hline \multicolumn{7}{|l|}{ Tumor site } \\
\hline Colon & 29 & $18(62.1 \%)$ & 44.8 & & 1 & \\
\hline Rectal & 25 & $20(80.0 \%)$ & 37.7 & 0.139 & $1.08(0.55-2.12)$ & 0.832 \\
\hline \multicolumn{7}{|l|}{ Tumor grade } \\
\hline Low + Intermediate & 39 & $28(71.8 \%)$ & 40.1 & & 1 & \\
\hline High & 15 & $10(66.7 \%)$ & 42.4 & 0.878 & $1.01(0.44-2.32)$ & 0.989 \\
\hline \multicolumn{7}{|l|}{ Duke's stage } \\
\hline$A+B$ & 15 & $7(46.7 \%)$ & $24.2^{\mathrm{a}}$ & & 1 & \\
\hline$C+D$ & 39 & $31(79.5 \%)$ & 39.3 & 0.082 & $1.12(0.71-1.77)$ & 0.624 \\
\hline \multicolumn{7}{|c|}{ Mesothelin levels (ng/ml) } \\
\hline$\leq 36.50$ & 27 & $14(51.9 \%)$ & 58.2 & & 1 & \\
\hline$>36.50$ & 27 & $24(88.9 \%)$ & 30.7 & $<0.001$ & $4.43(1.93-10.15)$ & $<0.001$ \\
\hline
\end{tabular}

aean survival time was provided when median OS could not be calculated.

${ }^{\mathrm{b}} P$ value was adjusted by age and sex.

\section{Association of mesothelin levels with survival in colorectal cancer patients}

Next, we used log-rank test to analyze whether mesothelin level in plasma is associated with colorectal cancer patients' prognosis. As shown in Table 4, patients with lower mesothelin in plasma had longer survival time, compared with patients whose mesothelin levels were higher than $36.50 \mathrm{ng} / \mathrm{ml}$ (log-rank $P<0.001)$. Furthermore, cox regression analysis revealed that there were statistically significant associations between elder individuals, drinkers and lower survival time (adjusted $\mathrm{HR}=2.83$, 95\% CI $=1.33-6.00, P=0.007$; adjusted $\mathrm{HR}=3.72,95 \%$ $\mathrm{CI}=1.26-11.0, P=0.018)$. However, when stratified by sex, smoking status, tumor site, size and grade and Duke's stage, we observed no significant difference in survival time in each following subgroup compared with each above subgroup (log-rank $P>0.05$ ). We also found that patients with higher levels of plasma mesothelin had a prominent poor prognosis than those with lower mesothelin levels (adjusted HR = $4.43,95 \% \mathrm{CI}=1.93-10.15, P<0.001)$.

In addition, unadjusted survival curves demonstrated a significant difference in 5-year survival between higher and lower level of mesothelin in colorectal cancer patients. As shown in Figure 2, patients with high level of mesothelin had a worse survival than patients with lower level of mesothelin $(P=0.001)$.

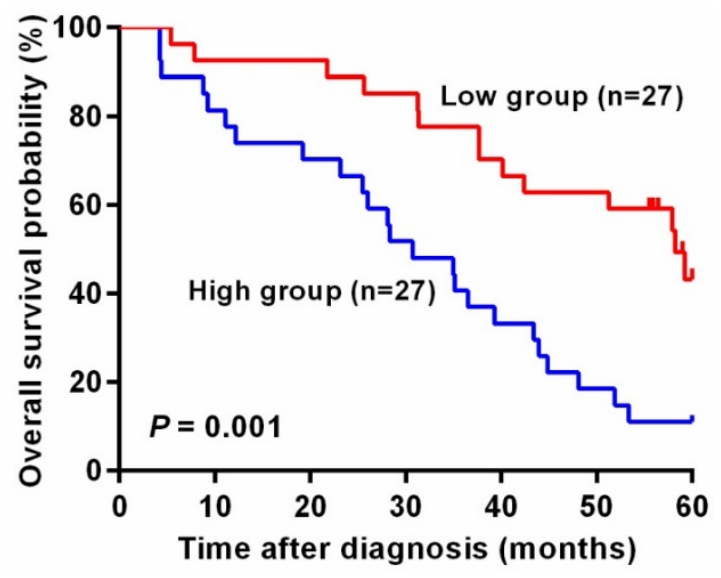

Figure 2. Kaplan-Meier curves of overall survival for plasma mesothelin levels in colorectal cancer patients. The cutoff value of plasma mesothelin was derived by Youden index $(36.50 \mathrm{ng} / \mathrm{ml})$. High group represents patients with plasma mesothelin higher than $36.50 \mathrm{ng} / \mathrm{ml}(\mathrm{n}=27)$ and Low group represents patients with plasma mesothelin lower than $36.50 \mathrm{ng} / \mathrm{ml}$ $(n=27)$.

\section{Discussion}

Previous studies have demonstrated that mesothelin can regulate growth and apoptosis in cancers [17]. Some studies suggested that mesothelin was associated with lymphatic invasion of colorectal cancer and over-expressed mesothelin in serum has potential ability to detect and screen colon cancer [14, 15]. Serum or blood mesothelin was suggested to be potential biomarker for early diagnosis or survival time in many cancers $[18,19]$. However, the utility of mesothelin as biomarker in colorectal cancer was 
unclear. As far as we know, this is the first study to investigate the potential association of plasma mesothelin with colorectal cancer diagnosis and survival.

In the present study, we estimated plasma mesothelin levels in 147 colorectal cancer cases and 121 healthy controls by using ELISA to evaluate whether mesothelin can be a diagnostic and prognostic biomarker in colorectal cancer. We found that individuals with colorectal cancer had a significantly increased mesothelin level (>36.50 $\mathrm{ng} / \mathrm{ml}$ ) in plasma than cancer-free group. We also displayed a significant trend of increased level of mesothelin with Duke's stage of plasma samples from stage A to D. Taken together, our results indicated that higher mesothelin level in plasma may contribute to the susceptibility or progression of colorectal cancer.

ROC analysis has revealed that the pooled sensitivity was only $48.3 \%$ although the specificity was $83.5 \%$. The AUC of 0.690 only touched a marginal clinical value in diagnosing colorectal cancer. Therefore, a larger cohort of prospective samples from additional hospitals need to be validated. Moreover, we only recruited the controls from those seeking medical care in local hospitals. We also did not recruit more appropriate control group, including some patients with other colorectal diseases. Due to these limitations of our study, the plasma mesothelin may has a moderate diagnosis value of colorectal cancer. In addition, a significant association of high mesothelin level in plasma with poor survival was confirmed in 54 colorectal cancer patients. We found that individuals with lower mesothelin level may have a remarkably higher survival rate than patients with higher mesothelin level. Our results, along with recent researches [20, 21], highlight the association of overexpressed with poor prognosis in several cancers. Together, it is possible to apply plasma mesothelin in diagnosis and prognosis of colorectal cancer. Recent studies have found that some potential biomarkers can be combined to apply in diagnosis or prognosis of cancers to enhance the sensitivity and specificity [22, 23], implying the diagnosis value of plasma mesothelin with some other biomarkers. Owning to the invasion of pathological diagnosis or colonoscopy to patients, we need to find some biomarkers more safe, effective and without surgery, like plasma biomarkers.

We also compared mesothelin level between preoperative and postoperative plasma samples. We found that mesothelin levels in plasma of colorectal cancer cases were slightly decreased 7 days after surgical tumor resection. In spite of no significant level change in 9 paired patients, further more paired plasma samples should be included to identify the association of mesothelin with the effect of tumor resection. However, the sample size of colorectal cancer cases was small and more paired samples need to be collected.

In conclusion, we have identified the plasma mesothelin as a potential biomarker with diagnosis and prognosis values in colorectal cancer, which was significantly increased in colorectal cancer cases, compared with controls. Further studies in biological function are needed to validate our findings.

\section{Supplementary Material}

Supplementary S1.

http://www.jcancer.org/v08p1355s1.pdf

\section{Acknowledgements}

This study was supported by National Natural Science Foundation of China (81373091), the Health Distinguished Young Scholars of Nanjing Municipal Science and Technology Commission (JQX13011), the Qinlan Project of Jiangsu (M. W.), and the Priority Academic Program Development of Jiangsu Higher Education Institutions (Public Health and Preventive Medicine).

\section{Competing Interests}

The authors have declared that no competing interest exists.

\section{References}

1. Ferlay J, Soerjomataram I, Dikshit R, et al. Cancer incidence and mortality worldwide: sources, methods and major patterns in GLOBOCAN 2012. Int J Cancer. 2015; 136: E359-86.

2. Siegel RL, Miller KD, Jemal A. Cancer statistics, 2016. CA Cancer J Clin. 2016; 66: 7-30.

3. Smith RA, Andrews K, Brooks D, et al. Cancer screening in the United States, 2016: A review of current American Cancer Society guidelines and current issues in cancer screening. CA Cancer J Clin. 2016; 66: 96-114.

4. Shaukat A, Mongin SJ, Geisser MS, et al. Long-term mortality after screening for colorectal cancer. N Engl J Med. 2013; 369: 1106-14.

5. Siegel R, DeSantis C, Virgo K, et al. Cancer treatment and survivorship statistics, 2012. CA Cancer J Clin. 2012; 62: 220-41.

6. Locker GY, Hamilton S, Harris J, et al. ASCO 2006 update of recommendations for the use of tumor markers in gastrointestinal cancer. J Clin Oncol. 2006; 24: 5313-27.

7. Chang K, Pastan I. Molecular cloning of mesothelin, a differentiation antigen present on mesothelium, mesotheliomas, and ovarian cancers. Proc Natl Acad Sci U S A. 1996; 93: 136-40.

8. Chang K, Pastan I, Willingham MC. Isolation and characterization of a monoclonal antibody, K1, reactive with ovarian cancers and normal mesothelium. Int J Cancer. 1992; 50: 373-81.

9. Creaney J, Yeoman D, Naumoff LK, et al. Soluble mesothelin in effusions: a useful tool for the diagnosis of malignant mesothelioma. Thorax. 2007; 62: 569-76.

10. Kendrick ZW, Firpo MA, Repko RC, et al. Serum IGFBP2 and MSLN as diagnostic and prognostic biomarkers for pancreatic cancer. HPB (Oxford). 2014; 16: 670-6.

11. Luo L, Shi HZ, Liang QL, et al. Diagnostic value of soluble mesothelin-related peptides for malignant mesothelioma: a meta-analysis. Respir Med. 2010; 104: 149-56.

12. Hassan R, Schweizer C, Lu KF, et al. Inhibition of mesothelin-CA-125 interaction in patients with mesothelioma by the anti-mesothelin monoclonal antibody MORAb-009: Implications for cancer therapy. Lung Cancer. 2010; 68: $455-9$.

13. Hassan R, Kreitman RJ, Pastan I, et al. Localization of mesothelin in epithelial ovarian cancer. Appl Immunohistochem Mol Morphol. 2005; 13: 243-7. 
14. Kawamata F, Homma S, Kamachi H, et al. C-ERC/mesothelin provokes lymphatic invasion of colorectal adenocarcinoma. J Gastroenterol. 2014; 49: 81-92.

15. Bostanci O, Kemik O, Kemik A, et al. Preoperative serum levels of mesothelin in patients with colon cancer. Dis Markers. 2014; 2014: 161954.

16. Ruopp MD, Perkins NJ, Whitcomb BW, et al. Youden Index and optimal cut-point estimated from observations affected by a lower limit of detection. Biom J. 2008; 50: 419-30.

17. Zheng C, Jia W, Tang Y, et al. Mesothelin regulates growth and apoptosis in pancreatic cancer cells through p53-dependent and -independent signal pathway. J Exp Clin Cancer Res. 2012; 31: 84

18. Huang CY, Cheng WF, Lee CN, et al. Serum mesothelin in epithelial ovarian carcinoma: a new screening marker and prognostic factor. Anticancer Res. 2006; 26: 4721-8.

19. Aktas B, Kasimir-Bauer S, Wimberger P, et al. Utility of mesothelin, L1CAM and Afamin as biomarkers in primary ovarian cancer. Anticancer Res. 2013; 33: 329-36.

20. Einama T, Homma S, Kamachi $\mathrm{H}$, et al. Luminal membrane expression of mesothelin is a prominent poor prognostic factor for gastric cancer. Br J Cancer. 2012; 107: 137-42.

21. Thomas A, Chen Y, Steinberg SM, et al. High mesothelin expression in advanced lung adenocarcinoma is associated with KRAS mutations and a poor prognosis. Oncotarget. 2015; 6: 11694-703.

22. Zhang $W$, Zheng $X$, Wang $X$. Oxidative stress measured by thioredoxin reductase level as potential biomarker for prostate cancer. Am J Cancer Res. 2015; 5: 2788-98.

23. Zheng D, Zhang J, Ni J, et al. Small nucleolar RNA 78 promotes the tumorigenesis in non-small cell lung cancer. J Exp Clin Cancer Res. 2015; 34: 49. 